\title{
Rapid discriminated bar-press avoidance through avoidance shaping
}

\author{
J. D. KEEHN AND C. D. WEBSTER \\ ALCOHOLISM AND DRUG ADDICTION RESEARCH FOUNDATION, TORONTO
}

Intensive work with individual rats showed that discriminated avoidance bar-pressing behavior is acquired within a single session when this response is shaped directly as an avoidance response.

A discriminated avoidance training procedure which is normally adequate for establishing running responses in the rat is completely unsuitable for training these animals to press a bar (D'Amato \& Schiff, 1964; Meyer, Cho, \& Wesemann, 1960). This procedure involves presenting a "warning" signal a few seconds prior to escapable shock, and allowing a response in the signal-shock interval to terminate the signal and avoid shock. When running is the response, responses occur after shock onset (escape responses) early in training, but before shock onset (avoidance responses) later on; the response moves forward in time Pavlovian-wise. When bar pressing is the response, the transition from escape to avoidance responding rarely occurs. It does, however, if pulsed instead of continuous shock is used (D'Amato, Keller, \& DiCara, 1964; Hurwitz, 1964). This could happen not because pulsed shock elicits anticipatory escape responses more effectively than does continuous shock, but because with pulsed shock the likelihood of responses occurring in the absence of shock (avoldance responses) is high, even when these responses do not actually anticipate the beginning of a series of shock pulses. That is, the advantage of the pulsed-shock technique may be that it provides conditions favoring the emission of bar presses reinforced by shock avoidance (Keehn, 1966) instead of by shock termination.
These and other considerations suggest that the practice of shaping escape responses and then testing the extent to which anticipatory avoidance responses occur might be a less effective method of establishing discriminated avoidance bar pressing in the rat than the tactic of shaping avoidance bar presses directly by omitting shocks when approximate responses occur. This latter tactic was adopted in the present study, in which the practicability of several avoidance shaping procedures was examined by intensive study of single animals.

Subjects

Several miscellaneous white and hooded rats were used. Of those reported below, C3, K2, K4, and K5 were naive albinos, about 100 days old. Animals $\mathrm{SH}, \mathrm{SM}, \mathrm{SQ}$, and SZ had previously been given saline injections as controls for another experiment.

\section{Apparatus}

A Grason-Stadler two bar rat chamber Type E3125D was used. For Animal C3 the standard left bar was used and the right bar was removed. For the other animals the left bar was removed and the right bar was replaced by a Hawley retractable lever Type HR121. This bar was $1-1 / 2$ in. wide; its facility for retraction was not used. Warning stimuli were combinations of the standard chamber stimulus lights (with house light off) with either a buzzer or a white noise. The buzzer was homemade and raised the ambient noise from 88 to $99 \mathrm{~dB}$. The white noise was obtained from a Grason-Stadler 901B generator; it raised the ambient noise level to $93 \mathrm{~dB}$. A GrasonStadler shock generator Type E1064GS was used to

Table 1. Procedural details of avoidance shaping and testing

\begin{tabular}{lrrrrrrrr} 
& K2 & K4 & K5 & SH & SM & SQ & SZ & C3 \\
Bar weight (g) & 2 & 6 & 6 & 6 & 6 & 6 & 6 & 20 \\
Signal: Light & + & + & + & + & + & + & + & + \\
$\quad$ Buzzer & + & + & - & - & - & - & - & - \\
$\quad$ Noise & - & - & + & + & + & + & + & + \\
Shock: Intensity (mA) & 1.0 & 1.0 & 1.0 & .3 & .4 & .5 & .5 & .5 \\
$\quad$ Duration (sec) & .5 & .5 & .5 & ESC. & ESC. & ESC. $/ 2$ & ESC. & ESC. \\
$\quad$ Interval (sec) & 10 & 10 & 10 & - & - & 2 & - & - \\
Intervals: Response-signal & 20 & 10 & 10 & 30 & 30 & 30 & 30 & VI30 \\
$\quad$ Signal-shock & 20 & 15 & 15 & 7 & 5 & 5 & 5 & 5 \\
Shaping: Time (min) & 120 & 95 & 195 & 48 & 40 & 54 & 105 & 100 \\
$\quad$ Trials & 228 & 286 & 589 & 70 & 60 & 60 & 106 & 120 \\
$\quad$ Shocks & 48 & 21 & 183 & 27 & 28 & 58 & 55 & 77 \\
$\quad$ Criterion & $10 / 10$ & $10 / 10$ & $10 / 10$ & TRIALS & TRIALS TRIALS TIME & $7 / 10$ \\
\hline TEST SHOCK: DURATION & .5 & .5 & .5 & .2 & .2 & .2 & .2 & ESCAPF \\
$\quad$ INTERVAL & 10 & 10 & 10 & 2 & 2 & 2 & 2 & - \\
\hline
\end{tabular}




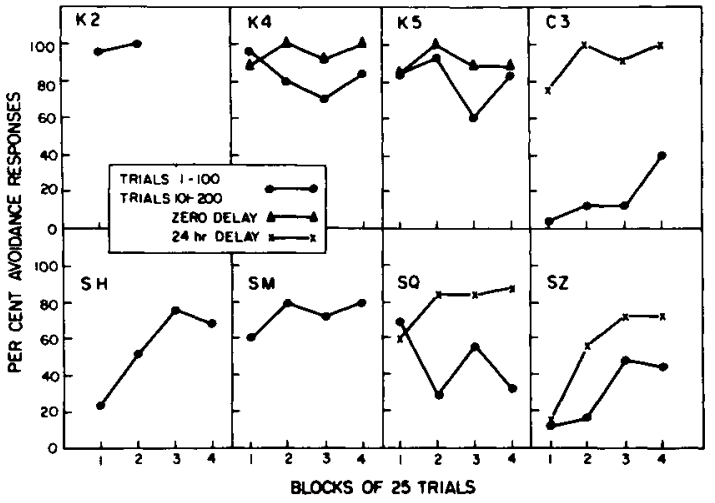

Fig. 1. Percentage avoidance responses by individual animals over blocks of 25 trials after avoidance shaping.

deliver scrambled shocks to the grid floor of the experimental chamber. Data were collected on cumulative recorders and magnetic counters.

Procedure

Procedural details are shown in Table 1. In all cases the signal preceded programmed shock by the signal-shock interval, and was terminated by either approximate or actual avoidance responses (K2, K4, K5), or by actual or approximate escape or avoidance responses (SH, SM, SQ, SZ, C3). Animal $\mathrm{SQ}$ was trained and tested according to the procedure described by D'Amato, Fazzaro, \& Etkin (1967); Animals SH, SM, and SZ were tested with this procedure but were trained with avoidance shaping and continuous escapable shock rather than with pulsed shock. Animals K2, K4, K5, and C3 were "avoidance shaped" to the designated criterion performance of avoidance responding and then tested unaided for 100 trials (50 for $\mathrm{K} 2$ through apparatus failure) with conditions otherwise unchanged. $\mathrm{K} 4$ and $\mathrm{K} 5$ continued for a further 100 trials; C3 was given a second 100 trials a day later. Similar tactics were adopted with the other $\mathrm{Ss}$, except that the criteria for ending shaping were different, and the method of shock delivery changed from training to testing, as shown in Table 1. In all cases shock intensities and frequencies were arranged so as not to establish bar holding or freezing behavior.

\section{Results and Discussion}

Percentages of avoidance responses, in blocks of 25 trials, over 100 trials immediately after shaping, and over 100 further trials either directly after the foregoing, or after a day's delay, are shown in Fig. 1. The highest level of avoidance responding was es- tablished in $\mathrm{K} 2, \mathrm{~K} 4$, and $\mathrm{K} 5$. However, these animals were not tested until the avoidance response was well acquired, and more time and trials were invested in manually training these animals than in training the others. This was necessary because it was often difficult to hold the animals in the vicinity of the bar with brief shocks spaced 10 sec apart, and because it was difficult to shape the animals beyond the stage of almost, but not quite, pressing the bar.

These difficulties were easily overcome when bar pressing was shaped first with escapable and then with pulsed shock. The former ensured that bar presses occurred quickly after shock onset, and the latter increased the probability of bar presses in the absence of shock. These latter, avoidance, bar presses were not just escape bar presses moved forward in time, for they still required shaping after the escape response was acquired (cf., D'Amato et al, 1967). We actually engaged in very little shaping after the introduction of pulsed shock (none with SH, SM, or SZ; 22 trials with SQ), which is probably why these animals were less proficient avoiders in the first 100 test trials than were $\mathrm{K} 2, \mathrm{~K} 4$, and $\mathrm{K} 5$.

Our experiment fails to support Meyer, Cho, \& Wesemann's (1960) suggestion that discriminated avoidance bar pressing is just not a suitable response for studying in the rat. In attempting to shape this response we quickly obtained avoidance running and "begging" responses and it soon became plain that discriminated avoidance bar pressing is more difficult to train than these other responses simply because it is a topographically more involved response. If the response is shaped, and shaped as an avoidance response (Keehn, 1966), it can be established in the rat by normal operant conditioning procedures.

\section{References}

D'AMATO, M. R., FAZZARO, J., \& ETKIN, M. Discriminated barpress avoidance maintenance and extinction in rats as a function of shock intensity. J. comp. physiol. Psychol., 1967, 63, 351-354.

D'AMATO, M. R., KELLER, D., \& DICARA, L. Facilitation of discriminated avoidance learning by discontinuous shock. J. comp. physiol Psychol, 1964, 58, 344349.

D'AMATO, M. R., \& SCHIFF, D. Long-term discriminated avoidance performance in the rat. J. comp. physiol. Psychol, 1964, 57, 123-126.

HURWITZ, H. M. B. Method for discriminative avoidance training. Science, 1964, 145, 1070-1071.

KEEHN, J. D. Avoidance responses as discriminated operants. Brit. J. Psychol, 1966, 57, 375-380.

MEYER, D. R., CHO, C., \& WESEMANN, A. S. On problems of conditioning discriminated lever-press avoidance responses. Psychol Rev., $1960,67,224-228$. 